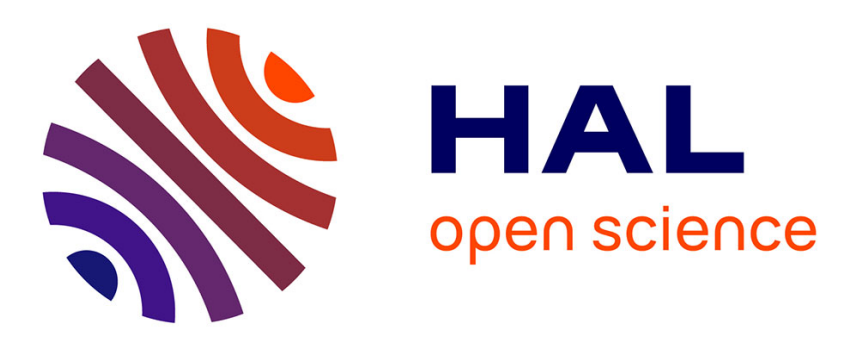

\title{
Joint BER Optimization and Blind PAPR Reduction of OFDM Systems with Signal Space Diversity
}

\author{
Tarak Arbi, Benoit Geller
}

\section{To cite this version:}

Tarak Arbi, Benoit Geller. Joint BER Optimization and Blind PAPR Reduction of OFDM Systems with Signal Space Diversity. IEEE Communications Letters, 2019, 10.1109/LCOMM.2019.2931898 . hal-02906498

\section{HAL Id: hal-02906498 \\ https://hal.science/hal-02906498}

Submitted on 24 Jul 2020

HAL is a multi-disciplinary open access archive for the deposit and dissemination of scientific research documents, whether they are published or not. The documents may come from teaching and research institutions in France or abroad, or from public or private research centers.
L'archive ouverte pluridisciplinaire HAL, est destinée au dépôt et à la diffusion de documents scientifiques de niveau recherche, publiés ou non, émanant des établissements d'enseignement et de recherche français ou étrangers, des laboratoires publics ou privés. 


\title{
Joint BER Optimization and Blind PAPR Reduction of OFDM Systems with Signal Space Diversity
}

\author{
Tarak Arbi and Benoit Geller
}

\begin{abstract}
This letter deals with a Peak-to-Average Power Ratio (PAPR) reduction scheme for Orthogonal Frequency Division Multiplexing (OFDM) systems with Signal Space Diversity. An original algorithm for the selection of two Rotated and Cyclically Q-Delayed (RCQD) QAM constellations is proposed to jointly optimize the Bit Error Rate (BER) and the blind detection performance of a SeLected Mapping (SLM) technique. Simulation results show that the proposed method achieves a large PAPR reduction with no spectral spoilage, and also enhances the BER performance in the case of DVB-T2 over different channels.
\end{abstract}

Index Terms-Rotated and Cyclically Q-Delayed (RCQD), Peak-to-Average Power Ratio (PAPR), SeLected Mapping (SLM).

\section{INTRODUCTION}

In frequency selective fading channels, it is often convenient to use OFDM as this low-complexity modulation is spectrally efficient. This can be problematic as the High Power Amplifier (HPA) usually operates near its saturation region for efficiency reasons and the transmission may suffer from in-band distortion and out-of-band radiation.

To face this issue, several solutions have been proposed in the literature to reduce the PAPR [1]. They can generally be divided into three main categories: signal distortion techniques, coding techniques and probabilistic techniques. The most basic method of the signal distortion techniques is the clipping technique; it replaces the peaks of the OFDM signal over a given threshold by a predetermined value at the price of a BER degradation. Differently, the key idea of coding techniques consists in choosing a set of code words with a desirable PAPR; the input data bit blocks are then mapped into one of the pre-selected code words with a correction code such as block or cyclic codes, at the price of spectral efficiency degradation. Finally, several probabilistic techniques have been proposed in the literature such as Active Constellation Extension (ACE) [2], Tone Reservation (TR) [3] and SLM [4]. With the SLM method, the transmitter generates in parallel several OFDM symbols representing all the same block of symbols multiplied by different phase sequences and chooses for transmission the one with the lowest PAPR. Moreover, TR techniques sacrifice some given subcarriers so as to transmit an auxiliary signal obtained by iteratively minimizing the PAPR of the overall OFDM symbol. ACE methods map the symbols on the outer boundaries of the original constellation to arbitrary positions without reducing the minimum distance between the symbols;

Tarak Arbi and Benoit Geller are with Department U2IS, ENSTA Paris, Institut Polytechnique de Paris, 828 boulevard des Maréchaux, 91120 Palaiseau, France (e-mail: tarak.arbi,benoit.geller@ensta-paris.fr). hence, these new degrees of freedom are used to reduce the PAPR. The PAPR gain achieved by these methods does not come without a price which may be a loss of the spectral or power efficiency, or a higher complexity [1]. In particular, the most penalizing limitation of the ordinary SLM algorithm is its spectral loss since for each OFDM symbol, the index of the used phase sequence must be coded and sent to the receiver; several proposals [5], [6] have thus been suggested in the literature to consider this issue.

Nevertheless, many of these methods can not be applied by construction for RCQD signals; indeed, these rotated constellations can possibly be used in the DVB-T2 standard [7], as they allow to achieve better performance over fading channels thanks to an increased Signal Space Diversity (SSD). The main idea of RCQD [8] is to rotate the conventional QAM constellation in order to correlate the in-phase (I) and the quadrature $(\mathrm{Q})$ signals. The resulting $\mathrm{I} / \mathrm{Q}$ components are then sent separately on independent channels. The angle at which the constellation is rotated is a fundamental feature for the performance gain of the system. In the literature, optimum angles were found based on several criteria such as maximizing the product distance between components or minimizing the average symbol error rate $[8]-[10]$.

The DVB-T2 standard [7] provides a series of rotation angles and recommends to use one or two PAPR reduction algorithms simultaneously, (i.e. ACE and TR). However, ACE cannot be implemented with RCQD signals. In this letter, the problems of BER optimization and PAPR reduction for rotated constellations OFDM systems are tackled jointly. We propose some rotation angles that allow the design of an efficient Blind SLM algorithm to reduce the PAPR with good performance in terms of BER; to achieve this goal, the transmitter having at its disposal an alphabet of several possible phase sequences, first rotates the original data according to these sequences and then sends rotated symbols that have the lowest PAPR. Finally, the receiver is able to recover, with no spectral loss, the transmitted sequence of phases by using a high performance soft ML estimator.

The rest of this letter is structured as follows. Section II briefly sketches our proposal and discusses the system model. Section III presents the system performance analysis and details the proposed Blind SLM method. Some numerical results are presented in Section IV. Section V concludes the letter. 


\section{PRoposal OUTLINE AND SYSTEM MODEL}

Throughout this letter, uppercase boldface letters denote sequences of $N$ elements, where $N$ is the subcarriers number.

\section{A. The transmitter side}

A message frame of $N\left(\log _{2}(M)\right)$ bits is Gray-mapped into a series $\boldsymbol{S}=\{s(0), s(1) \cdots, s(N-1)\}$ of $M$-QAM complex symbols. The transmitter can choose among an alphabet of $\mathrm{D}$ possible N-length phase sequences: $\left\{\boldsymbol{\Phi}^{(d)}, d=0,1, \cdots, D-\right.$ $1\}$, where $\boldsymbol{\Phi}^{(d)}=\left\{\phi_{0}^{(d)}, \phi_{1}^{(d)}, \cdots, \phi_{N-1}^{(d)}\right\}$. Moreover, let $\Delta_{T}$ be an integer needed to cyclically delay the $\mathrm{Q}$ component. The transmitter limits the PAPR with the following algorithm:

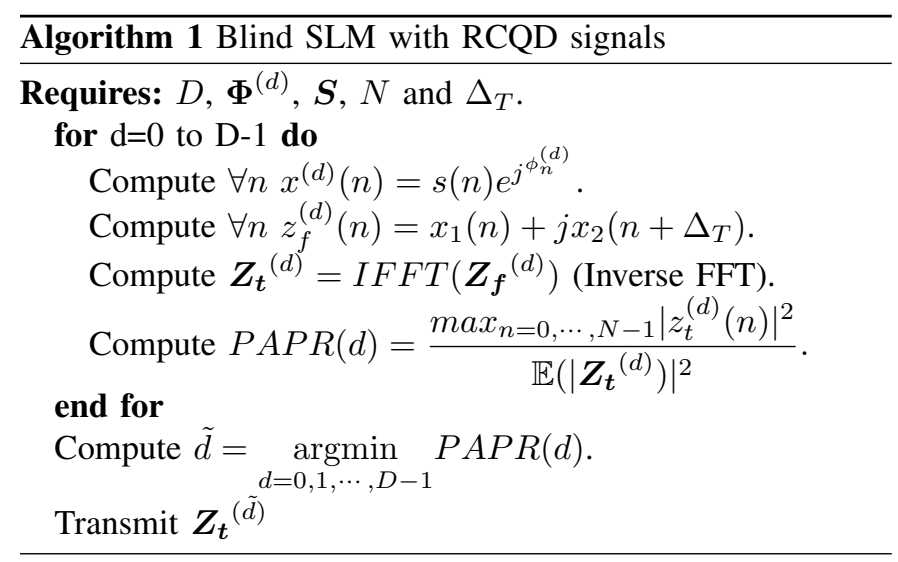

Note that in order to reduce the complexity at the receiver side, the number of possible rotation angles in the phase sequences is limited to two (i.e. $\left\{\phi_{n}^{(d)} \in\left\{\phi_{1}, \phi_{2}\right\}, \forall(d, n)\right\}$ ). Furthermore, to possibly avoid any prohibitive transmission delay, several IFFT blocks may be implemented in parallel and the algorithm may be stopped at a given satisfying threshold.

\section{B. The receiver side}

The receiver performs a Fast Fourier Transform (FFT) of the received signal and an inverse Q-delay to recover the received I/Q components of a symbol $x^{(d)}(n)$, which can be written as:

$$
\left\{\begin{array}{l}
y_{1}(n)=h_{1}(n) x_{1}^{(d)}(n)+v_{1}(n), \\
y_{2}(n)=h_{2}(n) x_{2}^{(d)}(n)+v_{2}(n),
\end{array}\right.
$$

where $h_{1}(n)$ and $h_{2}(n)$ are Rayleigh random variables and represent the channel attenuations at time $n$ and $n+D_{T}$ respectively, and $v(n)=v_{1}(n)+j v_{2}(n)$ is a complex additive noise, where $v_{1}(n)$ and $v_{2}(n)$ are independent zero-mean Gaussian variables of variance $N_{0}$. For a fading channel model with erasure events [7], if a component is erased the corresponding channel attenuation is equal to zero.

Finally, the ML receiver recovers the transmitted symbols such as:

$$
\hat{\boldsymbol{S}}=\underset{d=0,1, \cdots, D-1 \mid \boldsymbol{S} \in \mathbb{S}^{N}}{\operatorname{argmax}} P\left(\boldsymbol{Y} / \boldsymbol{\Phi}^{(d)}, \boldsymbol{S}, \boldsymbol{H}\right),
$$

where $\hat{\boldsymbol{S}}$ denotes the estimated symbols, $\mathbb{S}$ is the set of possible symbols and $\boldsymbol{Y}$ (resp. $\boldsymbol{H}$ ) refers to the received symbols (resp. the channel attenuations). Rotation angles $\phi_{1}$ and $\phi_{2}$ have a

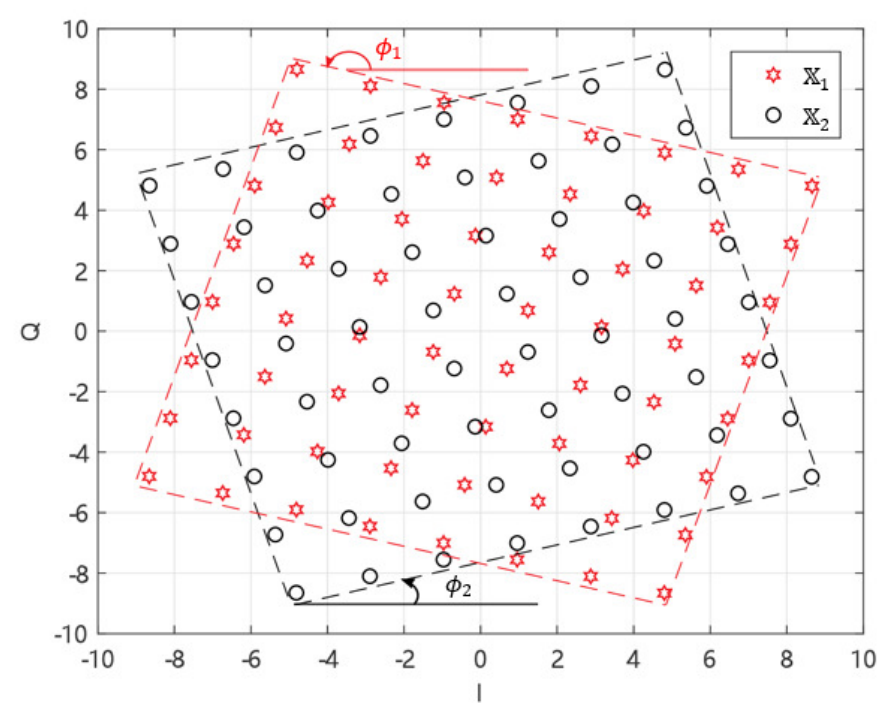

Fig. 1. Rotated constellations $\mathbb{X}_{1}$ and $\mathbb{X}_{2}$ for the 64-QAM signal

great influence on the receiver performance in (2) and must be carefully selected (see Fig. 1); the main purpose in the sequel of this letter is thus to investigate their impact on the overall performance and to propose a criterion to select these angles in section III.

\section{PERFORMANCE ANALYSIS AND PROPOSED BLIND SLM TECHNIQUE WITH SSD}

Performing the detection according to (2) is equivalent to the recovery of the phase sequence index using a soft ML algorithm followed by the separate estimation of the $N$ transmitted symbols. The following subsections study the probability of making an error at each of these two steps.

\section{A. Performance analysis}

1) Sequence Index Error Rate analysis: To reduce the Sequence Index Error Rate (SIER), for classical constellations, the Euclidean distance between points in $\mathbb{X}_{1}$ and in $\mathbb{X}_{2}$, where $\mathbb{X}_{i}$ is the rotated constellation obtained with the angle $\phi_{i}$ is maximized in [5]; however, by nature of the RCQD signal, this criterion is not effective [8]. Moreover, due to the high Signalto-Noise Ratio (SNR) approximation and the high kissing number [8], selecting angles by maximizing the so-called product distance may lead to sub-optimal performances. Instead, in order to minimize the SIER, we propose to minimize the average error probability $P_{\text {avg }}$ between points in constellations $\mathbb{X}_{1}$ and $\mathbb{X}_{2}$ (see Fig. 1):

$$
P_{a v g}\left(\phi_{1}, \phi_{2}\right)=\sum_{x \in \mathbb{X}_{1}} P(x) \sum_{\hat{x} \in \mathbb{X}_{2}} P(x \rightarrow \hat{x}) .
$$

$P(x \rightarrow \hat{x})$ can be expressed as:

$$
\begin{aligned}
P(x \rightarrow \hat{x})= & p^{2}+(1-p)^{2} P^{1,2}(x \rightarrow \hat{x}) \\
& +p(1-p) \sum_{i=1}^{2} P^{i}(x \rightarrow \hat{x}),
\end{aligned}
$$


where $P^{1,2}(x \rightarrow \hat{x})$ (resp. $\left.P^{i}(x \rightarrow \hat{x})\right)$ is the pairwise error probability when both components are received (resp. when only the $\mathrm{i}$-th component is received) and $p$ denotes the erasure probability.

Furthermore, similarly to [8], we obtain the following upper bounds:

$$
\begin{array}{r}
P^{1,2}(x \rightarrow \hat{x}) \leq \frac{1}{2} \prod_{i=1}^{2} \frac{1}{1+\frac{\left(x_{i}-\hat{x}_{i}\right)^{2}}{8 N_{0}}}, \\
P^{i}(x \rightarrow \hat{x}) \leq \frac{1}{2} \frac{1}{1+\frac{\left(x_{i}-\hat{x}_{i}\right)^{2}}{8 N_{0}}} .
\end{array}
$$

2) Bit error probability: Beyond phase sequence estimation, the angles choice also considerably influence the BER. Assuming a correct index estimation, the bit error probability can simply be developed as:

$$
P_{b}\left(\phi_{1}, \phi_{2}\right)=\sum_{i=1}^{2} P\left(\phi_{i}\right) P_{b}\left(\phi_{i}\right)
$$

where $P\left(\phi_{i}\right)$ and $P_{b}\left(\phi_{i}\right)$ are respectively the probability of using the angle $\phi_{i}$ and the bit error probability when the angle $\phi_{i}$ is chosen; we then have the following upper bound:

$$
P_{b}\left(\phi_{i}\right) \leq \frac{1}{\log _{2} M} \sum_{x \in \mathbb{X}_{i}} P(x) \sum_{\hat{x} \in \mathbb{X}_{i}} d_{H}(x, \hat{x}) P(x \rightarrow \hat{x}),
$$

where $P(x \rightarrow \hat{x})$ is given in (4)-(6) and $d_{H}(x, \hat{x})$ is the Hamming distance between the binary representation of symbols $x$ and $\hat{x}$. It is worth mentioning that differently from (3), as we aim at minimizing the BER for a given rotated constellation, $x$ and $\hat{x}$ now belong to the same rotated constellation $\mathbb{X}_{i}$ where $i$ can either be 1 or 2 .

\section{B. Proposed Blind SLM Technique with SSD}

1) RCQD constellations preselection: In order to design a robust system, the chosen couple of angles must drive to a low SIER, and several pairs of angles may satisfy this condition; thus, we propose to choose among the candidate rotation angles pairs, the couple which gives the best BER performance. This selection process can be formulated as:

$$
\left(\phi_{1}, \phi_{2}\right)=\underset{\left(\beta_{1}, \beta_{2}\right) \in \Sigma_{\epsilon}}{\operatorname{argmin}} P_{b}\left(\beta_{1}, \beta_{2}\right)
$$

where the set $\Sigma_{\epsilon}$ given by:

$$
\Sigma_{\epsilon}=\left\{\left(\beta_{1}, \beta_{2}\right) \in\right]-\pi, \pi\left[^{2} \mid P_{a v g}\left(\beta_{1}, \beta_{2}\right) \leq \epsilon\right\}
$$

must be as large as possible and $\epsilon$ refers to a threshold. A method to choose properly this threshold is discussed in section IV.

We finally mention that in order to generate an efficient PAPR reduction algorithm, the angles $\phi_{1}$ and $\phi_{2}$ should be quite different. Thus, in our simulations, the angles $\beta_{1}$ and $\beta_{2}$ that are separated by less than $\pi / 2$ were not considered in $\Sigma_{\epsilon}$.

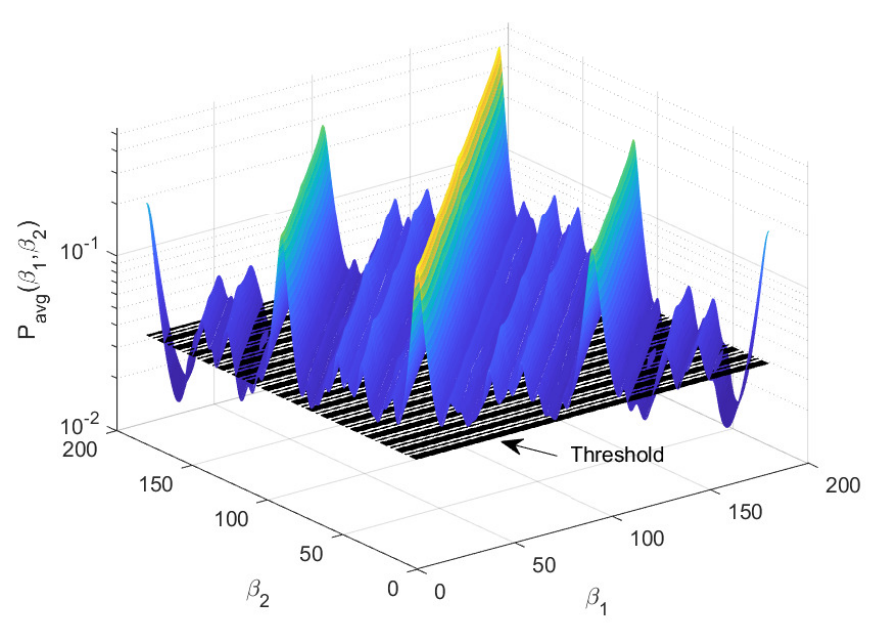

Fig. 2. $P_{\text {avg }}\left(\beta_{1}, \beta_{2}\right)$ for the 64-QAM constellation

2) Soft ML phase index estimator: Define the equally-balanced binary phase code sequence as $\boldsymbol{C}^{(d)}=\left\{c_{0}^{(d)}, c_{1}^{(d)} \cdots, c_{N-1}^{(d)}\right\}$ where $0 \leq d \leq D-1$ and:

$$
c_{n}^{(d)}=\left\{\begin{array}{l}
0 \text { if } \phi_{n}^{(d)}=\phi_{1} \\
1 \text { if } \phi_{n}^{(d)}=\phi_{2} .
\end{array}\right.
$$

Using the ML criterion, the receiver has to find the phase sequence such as:

$$
\hat{d}=\underset{0 \leq d \leq D-1}{\operatorname{argmax}} P\left(\boldsymbol{Y} \mid \boldsymbol{C}^{(d)}, \boldsymbol{H}\right) .
$$

Using the Max-Log approximation, we then obtain:

$$
\hat{d}=\underset{0 \leq d \leq D-1}{\operatorname{argmax}} \sum_{n=0}^{N-1} \Lambda_{n}^{c_{n}^{(d)}},
$$

where $\Lambda_{n}^{0}$ and $\Lambda_{n}^{1}$ are given by:

$$
\begin{aligned}
\Lambda_{n}^{0} & =\min _{a=a_{1}+j a_{2} \in \mathbb{X}_{1}}\left|y(n)-h_{1}(n) a_{1}-j h_{2}(n) a_{2}\right|^{2} \\
\Lambda_{n}^{1} & =\min _{a=a_{1}+j a_{2} \in \mathbb{X}_{2}}\left|y(n)-h_{1}(n) a_{1}-j h_{2}(n) a_{2}\right|^{2} .
\end{aligned}
$$

The ML estimator is theoretically based on an exhaustive search. The ML estimation thus requires at most $8 N M$ real multiplications and $N(6 M+D)-D$ real additions (see equations (13)-(15)). This complexity cannot be neglected for large constellations or/and subcarriers number; in this case, it is worth mentioning that in the literature several algorithms, such as the hard ML estimator [5] and a 2-step estimation technique based on a Viterbi algorithm followed by a verification and a correction step [11] have already been proposed and can be used to reduce the computational complexity of the blind SLM phase sequence estimation. As displayed in the following section, they allow to obtain similar BER performance when compared to the full-complexity ML estimation.

\section{NUMERICAL RESULTS}

\section{A. Angles preselection}

Fig. 2 presents results on $P_{a v g}\left(\beta_{1}, \beta_{2}\right)$ (see (3)) for the 64-QAM constellation at a reference $E_{b} / N_{0}=13 \mathrm{~dB}$. It 


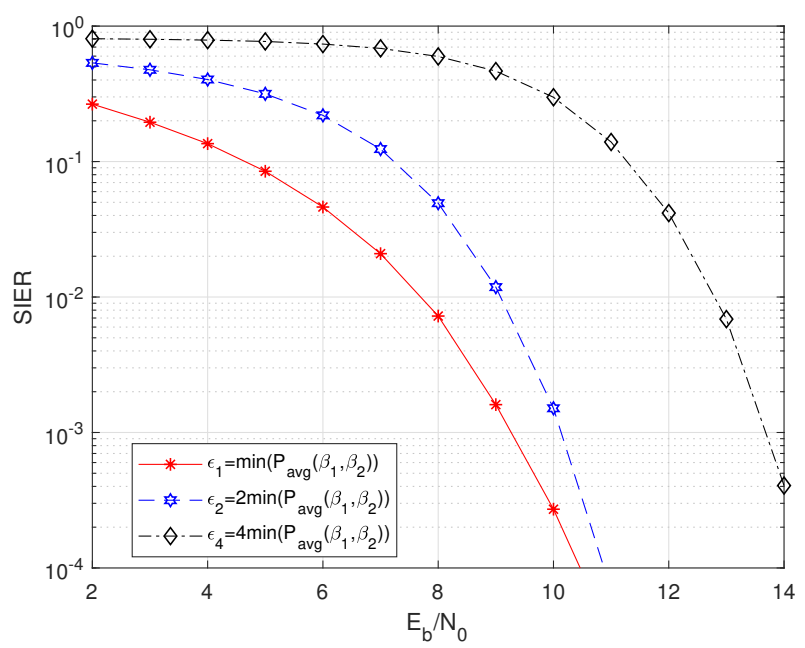

Fig. 3. SIER comparison at different thresholds for the 64-QAM over the Rayleigh fading channel without any erasure

TABLE I

PROPOSED ANGLES FOR THE RAYLEIGH FADING CHANNEL WITHOUT ERASURE

\begin{tabular}{|c|c|c|c|c|}
\hline & 4-QAM & 16-QAM & 64-QAM & 256-QAM \\
\hline$\phi_{1}$ & $27.5^{\circ}$ & $21.2^{\circ}$ & $15.5^{\circ}$ & $15.4^{\circ}$ \\
\hline$\phi_{2}$ & $-117.5^{\circ}$ & $159.1^{\circ}$ & $164.3^{\circ}$ & $-105.5^{\circ}$ \\
\hline$E_{b} / N_{0}$ & $6 \mathrm{~dB}$ & $9.5 \mathrm{~dB}$ & $13 \mathrm{~dB}$ & $17 \mathrm{~dB}$ \\
\hline
\end{tabular}

can be observed that several couples of angles achieve good performances, whereas numerous couples of rotation angles must be avoided. Hence a proper threshold in (10) should be carefully chosen. Fig. 3 compares, over the Rayleigh fading channel with $D=8$ and $N=256$, the SIER obtained through a Monte-Carlo simulation where $\hat{d}$ is evaluated with (13) for randomly selected angles verifying $P_{\text {avg }}\left(\beta_{1}, \beta_{2}\right)=\epsilon_{i}$ at the border of $\Sigma_{\epsilon_{i}}(i=1,2,4)$. It can be noticed that, among the considered thresholds, only $\epsilon_{1}=\min \left(P_{\text {avg }}\left(\beta_{1}, \beta_{2}\right)\right)$ and $\epsilon_{2}=2 \min \left(P_{\text {avg }}\left(\beta_{1}, \beta_{2}\right)\right)$ lead to a low SIER, for a system operating at $E_{b} / N_{0}=13 \mathrm{~dB}$. Therefore, one should rather choose the threshold $\epsilon_{2}$ as it allows to obtain a larger set $\Sigma_{\epsilon_{2}}$ (see (10)) and thus a better BER performance. We then select with (9) the best couple of angles in terms of BER at the reference $E_{b} / N_{0}$ and this process can be realized for any constellation (see Tables I and II).

\section{B. System performance}

Fig. 4 makes a comparison between the proposed method and the case where the receiver has an ideal Receiver Side Information (RSI) knowledge of the transmitter phase sequence

TABLE II

PROPOSED ANGLES FOR THE RAYLEIGH FADING CHANNEL WITH $15 \%$ OF ERASURE EVENTS

\begin{tabular}{|c|c|c|c|c|}
\hline & 4-QAM & 16-QAM & 64-QAM & 256-QAM \\
\hline$\phi_{1}$ & $25.7^{\circ}$ & $14.8^{\circ}$ & $6.3^{\circ}$ & $3.4^{\circ}$ \\
\hline$\phi_{2}$ & $-114.1^{\circ}$ & $169.9^{\circ}$ & $172.9^{\circ}$ & $176.3^{\circ}$ \\
\hline$E_{b} / N_{0}$ & $8.5 \mathrm{~dB}$ & $13.5 \mathrm{~dB}$ & $18.5 \mathrm{~dB}$ & $24 \mathrm{~dB}$ \\
\hline
\end{tabular}

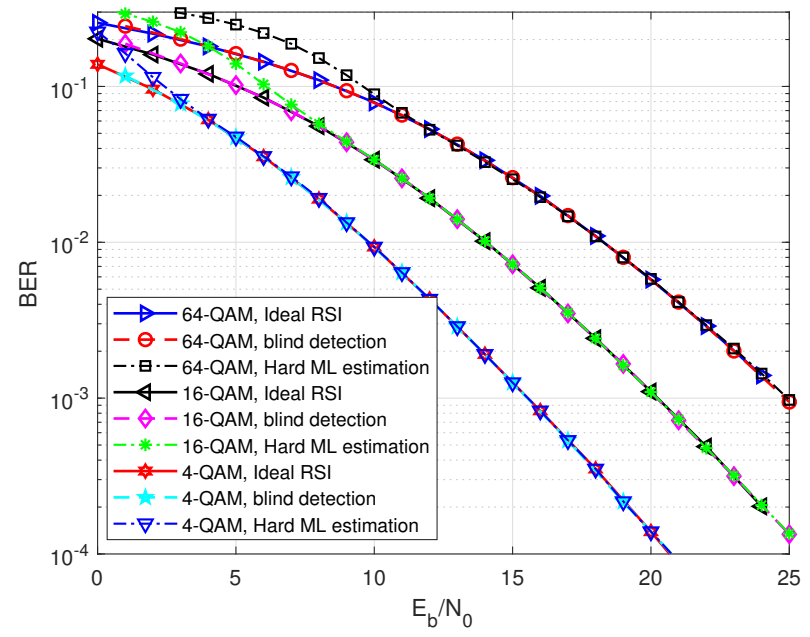

Fig. 4. BER comparison between the ideal RSI, the proposed method and the hard ML estimation over the Rayleigh channel without any erasure

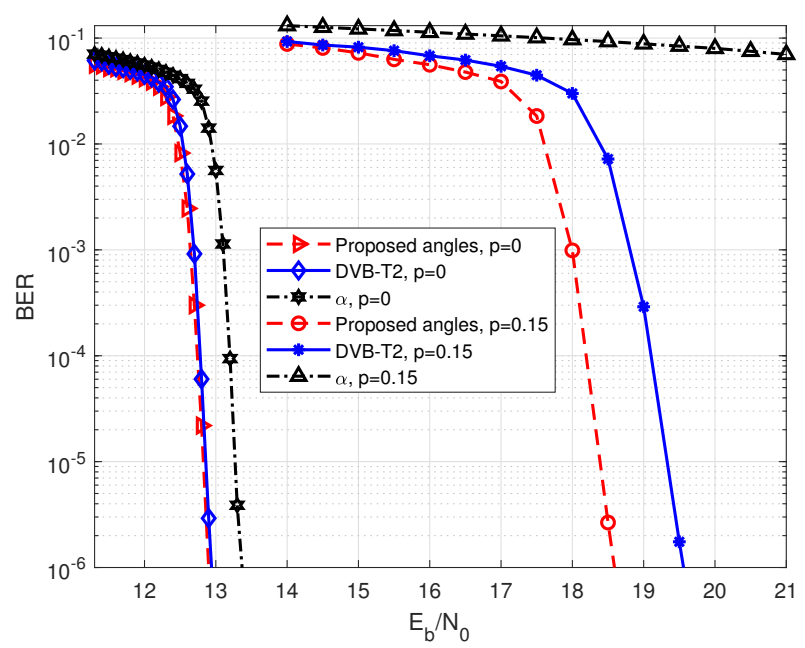

Fig. 5. BER comparison for the 64-QAM over Rayleigh fading channels with and without erasures

index, over the Rayleigh fading channel without erasure for $N=256$ and $D=8$ and for the angles chosen in Table I. It can be observed that there is roughly no difference between the soft ML estimator and the ideal method, which illustrates that our blind proposal avoids any index sequence transmission spoilage. Additionally, it can be observed that the low-complexity hard ML estimator [5] achieves, for moderate to high SNR, a near optimum performance. Furthermore, Fig. 5 compares the BER, obtained for the DVB-T2 [7] system with a 64800-bit long LDPC code of rate 4/5 with 25 -iteration min-sum decoding, between the proposed angles (see Tables I and II) and the angle currently used in DVB-T2 for 64-QAM. The performance of the proposed angles over Rayleigh fading channels without erasure is slightly better than that obtained with the angle currently used in DVB-T2. Moreover, for the Rayleigh fading channel with $15 \%$ of erasure, the proposed angle outperforms the BER obtained with the angle used in DVB-T2 by roughly $1 \mathrm{~dB}$. Similar results are obtained for 
other configuration parameters and other angles of the state of the art. In particular we find that at $B E R=10^{-6}$, our proposal achieves a $0.4 \mathrm{~dB}$ (resp. $7 \mathrm{~dB})$ gain in BER compared to the performance of the angles $\alpha=\operatorname{atan}\left(\frac{1 \pm \sqrt{5}}{2}\right)$ [8] over the Rayleigh channel without erasure (resp. with $15 \%$ of erasure).

Finally, Fig. 6 compares the PAPR reduction performance of the proposed algorithm with the clipping scheme (with preset clipping level at $75 \%$ of the maximum of the original OFDM symbols), the Tone Reservation algorithm defined in DVB-T2 [7] (with a clipping threshold of $7 \mathrm{~dB}$ and 10 iterations), and the theoretically optimal Partial Transmit Sequence (PTS) [12] (with $S=16$ sub-blocks and rotation angles $\{0, \pi\}$ ), for the 64-QAM constellation and $N=1024$.

First, we observe that the PAPR reduction performance of our proposal improves as $D$ increases (e.g. $3.7 \mathrm{~dB}$ gain with $D=16$ ). Furthermore, it can be observed that for any $D>2$, our proposal clearly achieves a better PAPR reduction than that of the TR algorithm.

The clipping scheme achieves a poorer PAPR reduction performance than that of our proposal for $D>4$. This performance could be improved with a lower clipping threshold; nevertheless, contrarily to our proposal, the associated BER performance would then be even more deteriorated.

Besides, when we combine the TR algorithm and our proposal for $\mathrm{D}=8$, we obtain roughly the same performance as the optimal PTS (about a $5 \mathrm{~dB}$ gain). However, the optimal PTS requires, in addition to a large side information, a high exponential complexity in $\mathrm{S}$ at the transmitter side; therefore, many extensions of the original PTS algorithm with suboptimal PAPR reduction performance have been proposed in the literature [13] but the associated PAPR reduction can seriously be degraded. In contrast to the PTS and the TR algorithms, besides that the complexity of our proposal is lower at the transmitter side but larger at the receiving end, our Blind SLM proposal avoids any spectral spoilage.

Finally, it must be enhanced that beyond the capability of the PAPR reduction itself, when choosing a PAPR reduction method, other criteria need to be considered in order to meet the requirements of the communication system, such as the BER performance, the spectral efficiency and the computational complexity [1]; by design, our proposal inherently takes into account all these issues: the BER is enhanced, there is no spectral loss and the PAPR improvement can be chosen according to a reasonable complexity trade-off at the receiver side.

\section{CONCLUSION}

An original method to reduce the PAPR is proposed for OFDM signals with rotated constellations. It is based on two sets of symbols belonging to two rotated constellations with two different angles. At the transmitting side, among several possible phase sequences, the best signal in terms of PAPR reduction is transmitted. To avoid any spectral efficiency loss, the receiver must estimate the transmitted phase sequence. Simulations results show the importance of the rotation angles choice and the efficiency of the detection algorithm. They

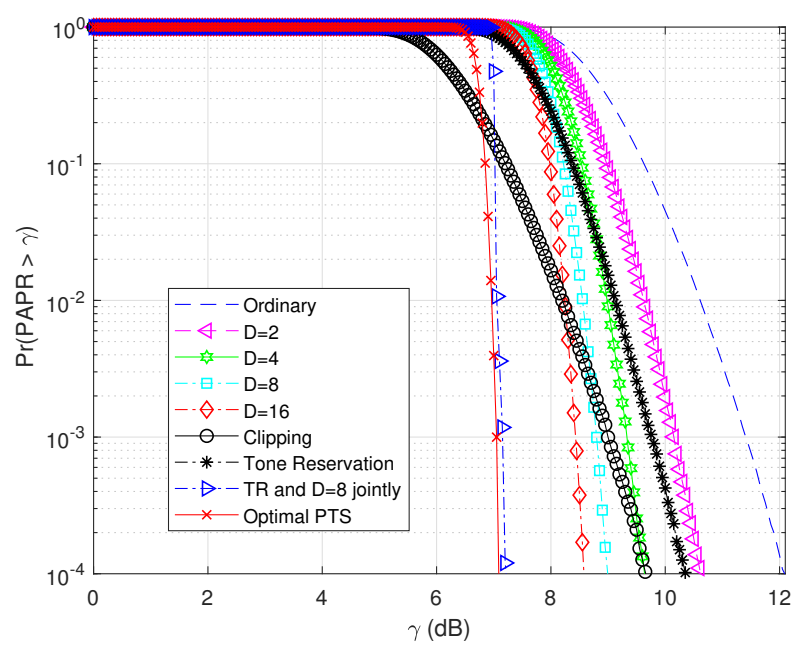

Fig. 6. PAPR reduction performance comparison between the considered methods for an OFDM system with 1024 subcarriers and the RCQD 64-QAM

also underline the great impact of the proposed method on the reduction of the PAPR, as well as the BER improvement.

\section{REFERENCES}

[1] T. Jiang and Y. Wu, "An Overview: Peak-to-Average Power Ratio Reduction Techniques for OFDM Signals," IEEE Transactions on Broadcasting, 54(2): 257-268, June 2008.

[2] K. Bae, J. G. Andrews, and E. J. Powers, "Adaptive active constellation extension algorithm for peak-to-average ratio reduction in OFDM,"in IEEE Communications Letters, 14(1): 39-41, Jan. 2010.

[3] S. S. K. C. Bulusu, M. Crussière, J. F. Hélard, R. Mounzer, Y. Nasser, O. Rousset, and A. Untersee, "Quasi-Optimal Tone Reservation PAPR Reduction Algorithm for Next Generation Broadcasting Systems: A Performance/Complexity/Latency Tradeoff With Testbed Implementation," IEEE Trans. on Broadcasting, 64(4): 883-899, Dec. 2018.

[4] S. J. Heo, H. S. Noh, J. S. No, and D. J. Shin, "A modified SLM scheme with low complexity for PAPR reduction of OFDM systems," IEEE Trans. on Broadcasting, 53(4): 804-808, Dec. 2007.

[5] M. Sghaier, F. Abdelkefi, and M. Siala, "Efficient embedded signaling through rotated modulation constellations for SLM-based OFDM systems", IEEE Inter. Conf. on Comm. (ICC), 5252-5256, June 2013.

[6] J. Ji, G. Ren, and H. Zhang, "A Semi-Blind SLM Scheme for PAPR Reduction in OFDM Systems With Low-Complexity Transceiver," IEEE Trans. on Vehicular Technology, 64(6): 2698-2703, June 2015.

[7] Implementation guidelines for a second generation digital terrestrial television broadcasting system (DVB-T2), European Telecom. Standard Institute ETSI TR 102831 V1.1.1, Oct. 2010.

[8] J. Boutros and E. Viterbo, "Signal space diversity: a power- and bandwidth-efficient diversity technique for the Rayleigh fading channel", IEEE Trans. on Inform. Theory, 44(4): 1453-1467, July 1998.

[9] Q. Xie, J. Song, K. Peng, and F. Yang, "Coded modulation with signal space diversity," IEEE Trans. on Wireless Communication, 10(2):660669, Feb. 2011.

[10] A. Abdmouleh, E. Boutillon, L. Conde-Canencia, C. A. Nour, and C. Douillard, "On Signal Space Diversity for non binary coded modulation schemes," IEEE 23rd Inter. Confer. on Telecom. (ICT), Greece, 1-5, 2016.

[11] A. Boonkajay and F. Adachi, "2-Step phase rotation estimation for LowPAPR signal transmission using blind selected mapping," 28th Annual Inter. Sym. on Personal, Indoor, and Mobile Radio Comm. (PIMRC), Montreal, 2017, pp. 1-5.

[12] Y. A. Jawhar, L. Audah, M. A. Taher, K. N. Ramli, N. S. M. Shah, M. Musa, and M. S. Ahmed, "A Review of Partial Transmit Sequence for PAPR Reduction in the OFDM Systems," IEEE Access, 7, pp. 18021$18041,2019$.

[13] S. Ku, C. Wang and C. Chen, "A Reduced-Complexity PTS-Based PAPR Reduction Scheme for OFDM Systems," in IEEE Transactions on Wireless Communications, 9(8), pp. 2455-2460, August 2010. 